\title{
Moving Antibiotic Stewardship from Theory to Practice
}

\author{
Payal K. Patel, MD, MPH ${ }^{1} 2^{*}$, Arjun Srinivasan, $\mathrm{MD}^{3}$
}

${ }^{1}$ Department of Internal Medicine, Division of Infectious Diseases, University of Michigan Medical School, Ann Arbor, MI; ${ }^{2}$ Department of Internal Medicine, Division of Infectious Diseases, VA Ann Arbor Healthcare System, Ann Arbor, MI; ${ }^{3}$ Division of Healthcare Quality Promotion, Centers for Disease Control and Prevention, Atlanta, GA.

We both attend on the Infectious Disease consult team in Veterans Affairs (VA) Hospitals, and predictably the conversation on afternoon rounds often revolves around antibiotics. When we have those discussions, our focus is not on a need to "preserve antibiotics" so they might be available to some unknown patient in the future. Rather, we are working with the primary team to provide the very best treatment for the patient entrusted to our care in the bed right in front of us. We believe it is in this context-providing optimal patient care-that the current efforts in the United States to improve antibiotic use should be viewed.

The growing challenges posed by antibiotic-resistant infections and the related threat of Clostridium difficile infection combine to sicken more than 2 million people each year and contribute to the deaths of more than 25,000 patients. ${ }^{1}$ Improving antibiotic use through antibiotic stewardship is often proposed to hospitalists as an important part of stemming this tide. While this is true, even as infectious disease specialists with strong interests in antimicrobial stewardship we do not find that pitch compelling when we are on clinical service.

What motivates us to optimize antibiotic use for our patients is the evidence that doing so will have direct and immediate benefits to the patients under our care. Improving antibiotic use has been proven to decrease a patient's risk of acquiring C. difficile infection or an antibiotic-resistant infection not at some ill-defined time in the future, but during their current hospital stay. ${ }^{2,3}$ Even more important, support from antibiotic stewardship programs has been proven to improve infection cure rates and reduce the risk of treatment failure for hospitalized patients. ${ }^{4}$ The bottom line of antibiotic stewardship is better patient care. Sometimes that means narrowing or stopping antibiotics to reduce the risks of adverse events. In other cases, like in the treatment of suspected sepsis, it means ensuring patients get broad spectrum antibiotics quickly.

The patient care benefits of improving antibiotic use led the Centers for Disease Control and Prevention (CDC)

\footnotetext{
*Address for correspondence and reprint requests: Payal K. Patel, MD, MPH, University of Michigan, Infectious Disease Clinic, Taubman Center, Floor 3 Reception D, 1500 East Medical Center Drive, SPC 5352, Ann Arbor, Ml 48109; Telephone: 734-845-5695; Fax: 734-845-3290; E-mail: payalkp@umich.edu
}

Received: February 17, 2017; Accepted: February 25, 2017

2017 Society of Hospital Medicine DOI 10.12788/jhm.2741 to issue a call in 2014 for all hospitals to have antibiotic stewardship programs, and to the development of The Core Elements of Hospital Antibiotic Stewardship Programs to support that effort. As of January 1, 2017, antibiotic stewardship programs that incorporate all the CDC core elements became an accreditation requirement of The Joint Commission, and the Centers for Medicare and Medicaid Services has proposed making the same requirement of all hospitals that participate in their payment programs.

This means the question is no longer whether we should have antibiotic stewardship efforts in hospitals, but how we can do this most effectively. As the physicians who provide the most care in hospitals, hospitalists are best positioned to turn stewardship theories into practice. The article from Graber et $a .^{5}$ in this issue of the Journal of Hospital Medicine provides some important information that can help busy hospitalists incorporate stewardship into daily practice. The authors reviewed their experience with implementing stewardship efforts in VA hospitals to see which specific interventions were most likely to translate into improved antibiotic use. Based on their findings, we offer some suggestions for three conditions: pneumonia, urinary tract infection (UTI), and skin and soft tissue infection (SSTI). Together, these conditions drive roughly two-thirds of all antibiotic use in US hospitals. ${ }^{6}$

\section{STEWARDSHIP IN PRACTICE: PNEUMONIA}

The literature on treatment of pneumonia is increasingly demonstrating that shorter use of antibiotics is often better. ${ }^{7}$ Even though current guidelines recommend 5 to 7 days of antibiotics for uncomplicated community-acquired pneumonia, average durations of therapy are often longer. ${ }^{8}$ Previous work published in the Journal of Hospital Medicine focused on improving antimicrobial documentation as well as access to local clinical guidelines and implementing a 72 hour antimicrobial "time out" by hospitalists. ${ }^{9}$ When these multimodal interventions tailored for hospitalists were in place, utilization of antibiotics improved. Graber et al..$^{5}$ also found that facility educational programs for prudent antimicrobial use and frequency of de-escalation review were associated with decreased overall antimicrobial use. Providing vague recommendations on antibiotic course, or none at all, at discharge or sign-out can lead to unnecessary antibiotics or an extended course of them. Pneumonia-specific interventions could target duration by outlining antibiotic course in hospitalist progress notes and at hand-off. 


\section{STEWARDSHIP IN PRACTICE: UTI}

Misuse of antibiotics in UTI often stems from overtreatment of asymptomatic bacteriuria or unneeded diagnostic testing. Often, the pivotal step in avoiding unnecessary treatment lies in the ordering of the urine culture. ${ }^{10}$ Graber et al. ${ }^{5}$ showed that order sets were associated with decreased antimicrobial use. In the case of UTI, hospitalists could work with the stewardship team to design order sets that guide providers to appropriate reasons for ordering a urine culture. Order sets could also help providers recognize important patient-specific risks for certain antibiotics, such as the risk of C. difficile with fluoroquinolones in an elderly patient. Targeting different steps in overutilization of antibiotics would encompass more prescribers and could lead to reducing other unnecessary testing, which is a current focus for many hospitalists.

\section{STEWARDSHIP IN PRACTICE: SSTI}

Skin and soft tissue infections (SSTI) also offer a specific disease state to use order sets and education to improve duration of antibiotics, decrease overuse of broad spectrum antibiotics, and reduce unnecessary diagnostic studies. For example, gram negative and/or anaerobic coverage are rarely indicated in treating SSTIs but are often used. SSTI-specific order sets and guidelines have already been shown to improve both diagnostic work-up and antibiotic treatment. ${ }^{11}$ As the providers who manage most of these infections in hospitals, hospitalists are ideally positioned to inform the development of SSTI order sets and pathways. The work by Graber et al. ${ }^{5}$ provides some important insights into how we can effectively implement interventions to improve antibiotic use. These insights have never been more important as more hospitals move toward starting or expanding antibi- otic stewardship programs. As leaders in patient safety and quality, and as the most important antibiotic prescribers in hospitals, hospitalists must play a central role in stewardship if we are to make meaningful progress.

Disclosure: Nothing to report.

\section{References}

1. Centers for Disease Control and Prevention. Antibiotic Resistance Threats in the United States, 2013. https://www.cdc.gov/drugresistance/pdf/ar-threats-2013-508. pdf. Accessed April 12, 2017.

2. Feazel LM, Malhotra A, Perencevich EN, Kaboli P, Diekema DJ, Schweizer ML. Effect of antibiotic stewardship programmes on Clostridium difficile incidence: a systematic review and meta-analysis. J Antimicrob Chemother. 2014;69(7):17481754.

3. Singh N, Rogers P, Atwood CW, Wagener MM, Yu VL. Short-course empiric antibiotic therapy for patients with pulmonary infiltrates in the intensive care unit. A proposed solution for indiscriminate antibiotic prescription. Am J Respir Crit Care Med. 2000;162(2 Pt 1):505-511.

4. Fishman N. Antimicrobial stewardship. Am J Med. 2006;119(6 Suppl 1):S53-S61; discussion S62-S70.

5. Graber CJ, Jones MM, Chou AF, et al. Association of inpatient antimicrobial utilization measures with antimicrobial stewardship activities and facility characteristics of Veterans Affairs medical centers. J Hosp Med. 2017;12:301-309.

6. Magill SS, Edwards JR, Beldavs ZG, et al. Prevalence of antimicrobial use in US acute care hospitals, May-September 2011. JAMA. 2014;312(14):1438-1446.

7. Viasus D, Vecino-Moreno M, De La Hoz JM, Carratala J. Antibiotic stewardship in community-acquired pneumonia. Expert Rev Anti Infect Ther. 2016:1-2019.

8. Avdic E, Cushinotto LA, Hughes AH, et al. Impact of an antimicrobial stewardship intervention on shortening the duration of therapy for community-acquired pneumonia. Clin Infect Dis. 2012;54(11):1581-1587.

9. Mack MR, Rohde JM, Jacobsen D, et al. Engaging hospitalists in antimicrobial stewardship: Lessons from a multihospital collaborative. J Hosp Med. 2016;11(8):576-580.

10. Trautner BW, Grigoryan L, Petersen NJ, et al. Effectiveness of an Antimicrobial Stewardship Approach for Urinary Catheter-Associated Asymptomatic Bacteriuria. JAMA Intern Med. 2015;175(7):1120-1127.

11. Jenkins TC, Knepper BC, Sabel AL, et al. Decreased antibiotic utilization after implementation of a guideline for inpatient cellulitis and cutaneous abscess. Arch Intern Med. 2011;171(12):1072-1079. 